\title{
More evidence for hidden spiral and bar features in bright early-type dwarf galaxies ${ }^{\star}$
}

\author{
F. D. Barazza ${ }^{1}$, B. Binggeli ${ }^{1}$, and H. Jerjen ${ }^{2}$ \\ 1 Astronomisches Institut, Universität Basel, Venusstrasse 7, 4102 Binningen, Switzerland \\ ${ }^{2}$ Research School of Astronomy and Astrophysics, The Australian National University, Mt Stromlo Observatory, Cotter Road, \\ Weston ACT 2611, Australia
}

Received 25 February 2002 / Accepted 31 May 2002

\begin{abstract}
Following the discovery of spiral structure in IC 3328 (Jerjen et al. 2000), we present further evidence that a sizable fraction of bright early-type dwarfs in the Virgo cluster are genuine disk galaxies, or are hosting a disk component. Among a sample of 23 nucleated dwarf ellipticals and dS0s observed with the Very Large Telescope in $B$ and $R$, we found another four systems exhibiting non-axisymmetric structures, such as a bar and/or spiral arms, indicative of a disk (IC 0783, IC 3349, NGC 4431, IC 3468). Particularly remarkable are the two-armed spiral pattern in IC 0783 and the bar and trailing arms in NGC 4431. For both galaxies the disk nature has recently been confirmed by a rotation velocity measurement (Simien \& Prugniel 2002). Our photometric search is based on a Fourier decomposition method and a specific version of unsharp masking. Some "early-type" dwarfs in the Virgo cluster seem to be former late-type galaxies which were transformed to early-type morphology, e.g. by "harassment", during their infall to the cluster, while maintaining part of their disk structure.
\end{abstract}

Key words. galaxies: general - galaxies: fundamental parameters - galaxies: photometry - galaxies: structure

\section{Introduction}

The physical nature and origin of early-type dwarf (essentially $\mathrm{dE}$ ) galaxies is still largely unknown (for a review see Ferguson \& Binggeli 1994). According to their apparent flattening, which is similar to the one measured for giant ellipticals (Binggeli \& Popescu 1995; Ryden \& Terndrup 1994), early-type dwarfs seem to be spheroids. This is supported by the fact that most of these systems are not rotation-supported (Ferguson \& Binggeli 1994; de Rijcke et al. 2001; Geha et al. 2001). On the other hand, it has always been suspected that a considerable number of early-type dwarfs might be disk galaxies. In fact, around 20 bright early-type dwarfs in the Virgo cluster are classified dS0 because of their S0-like morphology, exhibiting a lens, a bar, or high flattening (Sandage \& Binggeli 1984; Binggeli \& Cameron 1991). In certain evolutionary scenarios, like ram-pressure stripping (Gunn \& Gott 1972; Abadi et al. 1999) or galaxy harassment (Moore et al. 1998), dwarf ellipticals are believed to have originated from late-type spirals or irregulars, hence some of them might have retained their disk nature. In addition, Ryden et al. (1999) showed that many early-type dwarfs have "disky" isophotes, similar to giant ellipticals.

\footnotetext{
Send offprint requests to: F. D. Barazza, e-mail: barazza@astro.unibas.ch

* Based on observations collected at the European Southern Observatory, Chile.
}

Recently, Jerjen et al. (2000) discovered a weak spiral structure in the seemingly normal dwarf elliptical galaxy IC 3328 by means of deep VLT-photometry. This is unambiguous evidence for the presence of a disk in this galaxy, supporting the conjecture that the number of (hidden) disk galaxies among bright early-type dwarfs could be quite high.

Following the work of Jerjen et al. (2000), we carefully searched a larger sample of dEs observed with the VLT for additional indications of spiral or bar structure. We first applied the same techniques as for IC 3328, i.e. relying on residual images and Fourier analysis. Among the $23 \mathrm{dEs}$ studied we found seven promising candidates for hidden disk structure. However, when we further explored these objects we realized that the observed spiral and bar features which we took as disk signatures are accompanied, and could actually be caused, by a specific behaviour of the ellipticity and position angle profiles. By performing a set of simulations with artificial galaxies we convinced ourselves that the interplay between photometric parameters can indeed produce amazingly spiral-like twisting isophotes and thus mimic a genuine spiral structure. Hence, although the particular parameter combinations found in many galaxies are remarkable in themselves, they cannot unambiguously be interpreted as signs of disk structure.

Fortunately, the ambiguity can be solved by applying an unsharp masking technique, which is a model-free method to amplify local image residuals. Only four of the seven candidates mentioned (two of which are already classified as dS0), 
Table 1. Basic data of the early-type dwarfs considered in this study.

\begin{tabular}{lllcccccr}
\hline \hline VCC & Name & Type & $\begin{array}{l}R_{\mathrm{T}} \\
(1)\end{array}$ & $\begin{array}{c}M_{R_{\mathrm{T}}} \\
(2)\end{array}$ & $\begin{array}{c}B-R \\
(3)\end{array}$ & $\begin{array}{c}v_{\odot} \\
(4)\end{array}$ & \multicolumn{1}{c}{$\epsilon)$} & \multicolumn{1}{c}{ PA } \\
$(6)$ & \multicolumn{1}{c}{$(7)$} & \multicolumn{1}{c}{$(8)$} & \multicolumn{1}{c}{$(9)$} \\
\hline 0490 & IC 0783 & dS0(3), N & 12.63 & -18.52 & 1.34 & 1293 & 0.25 & 130 \\
0940 & IC 3349 & dE1, N & 13.56 & -17.59 & 1.25 & 1563 & 0.22 & 14 \\
1010 & NGC 4431 & dSO(5), N & 12.47 & -18.68 & 1.39 & 913 & 0.38 & 168 \\
1422 & IC 3468 & E1, N: & 12.64 & -18.51 & 1.16 & 1372 & 0.17 & 150 \\
\hline
\end{tabular}

in addition to IC 3328 (Jerjen et al. 2000), withstood the scrutiny of unsharp masking: IC 0783, IC 3349, NGC 4431, and IC 3468. These are the galaxies focused on in the present paper. Although none of the four discovered cases is as spectacular as IC 3328 with its tightly wound spiral, they show that the fraction of disk galaxies among bright early-type dwarfs, at least in the Virgo cluster, is $20 \%$ or larger. We recall that the situation is quite similar to classical (non-dwarf) elliptical galaxies, where also some Es turned out to be barred S0s, or to contain such a component, as betrayed by inner isophotal twists (Nieto et al. 1992).

The rest of the paper is organized as follows. In Sect. 2 we give some observational background and put this investigation into context with our more general project. In Sect. 3 we employ, and present the results of, a Fourier analysis of the galaxy images. Section 4 contains a brief account of the application of unsharp masking, which is the decisive detection tool. In Sect. 5 we discuss our findings and what they could mean, case by case. A summary is given in Sect. 6. Throughout this paper we assume a distance to the Virgo cluster of $D=17 \mathrm{Mpc}$, corresponding to $(m-M)=31.15$.

\section{Observational background}

This work is part of a larger project aimed at the determination of distances to dwarf elliptical galaxies in the Virgo and Fornax clusters by means of the Surface Brightness Fluctuations method, and a detailed photometric analysis of their brightness distributions. The galaxies were primarily selected by their morphological appearance, i.e. early-type dwarfs (dE, dS0), and by their apparent size, i.e. an isophotal radius $r_{B, 25}>30^{\prime \prime}$. So far, 25 objects have been observed in two runs at the Very Large Telescope at ESO Paranal Observatory in service mode. Details of the observations are to be reported elsewhere (Jerjen et al., in preparation). The important parameters of the four galaxies considered in this study are listed in Table 1 . The columns of the table are as follows: Cols. 1 and 2: identification of the observed galaxy; Col. 3: morphological type in the classification system of Sandage \& Binggeli (1984), taken from Binggeli et al. (1985); Col. 4: total $R$-band magnitude, corrected for galactic extinction (from Barazza et al., in preparation); Col. 5: absolute $R$-band magnitude; Col. 6: total colour index $B-R$; Col. 7: heliocentric radial velocity in $\mathrm{km} \mathrm{s}^{-1}$ (from the NED); Cols. 8 and 9: ellipticity $\epsilon=1-\frac{b}{a}$, where $a$ and $b$ are the major and minor axis, and position angle PA (from top counterclockwise), respectively, determined at an isophotal level of $\sim 25 \mathrm{mag} / \square^{\prime \prime}$ in $R$.

Direct $R$-band images of the galaxies are shown in the left column of Fig. 1. The image reduction and the determination of the standard photometric parameters have been performed with ESO's image processing package MIDAS. The detailed results of the photometry, like surface brightness profiles, effective radii, colour gradients etc., as well as best-fitting Sersic parameters will be presented elsewhere (Barazza et al., in preparation). Using the MIDAS application FIT/ELL3 we fitted ellipses to the isophotes of the galaxies and, hence, determined their centers, ellipticities and position angles. The ellipticities and position angles of the fitted ellipses versus the equivalent radius, $r=\sqrt{a b}$, are shown in the top two panels of Fig. 2. The variations of these two parameters are indicative of the asymmetric features we are interested in.

\section{Residual images and Fourier expansion}

In a first approach we subtracted the deprojected and azimuthally averaged light distribution of a galaxy from the original image. More precisely, we reconstructed a galaxy using the measured surface brightness profile with fixed ellipticity and position angle, as given in Cols. 8 and 9 of Table 1, and subtracted this model from the original. The results derived in this manner are shown in the second column of Fig. 1. Strong residuals are evident in the inner parts of all four galaxies.

IC 0783 and NGC 4431 show the most striking features; in both cases weak signs of spiral or bar structure can be discerned, or guessed, already in the direct images (left column). The spiral arms of IC 0783 are beautifully traced by the oscillations in the ellipticity and position angle profiles plotted in Fig. 2. The residual of IC 3349, which otherwise is a normal, almost round dwarf elliptical with a bright nucleus, shows an extended and seemingly lumpy structure along the major axis. The bright knot about $10^{\prime \prime}$ south-east of the center is caused by a luminous background galaxy. NGC 4431 is certainly the most interesting case. In the northern and southern part of its $R$-band image two dark arcs are visible (unfortunately barely so in the printed version). The residual shows that these arcs are at the end of a bar, forming the outer edges of a spiral-like feature, probably the trailing arms of the rotating bar.

Finally, in the residual of IC 3468 we note a prominent hourglass-like feature. This is in fact what is expected to appear whenever the ellipticity in the central part of a galaxy significantly changes with radius, and it was observed in more than these four galaxies (cf. Sect. 1). Indeed, by inspecting Fig. 2 (top row), we see that the ellipticity profile shows an inner peak in all four cases. Moreover, if an ellipticity change is combined with only a slight change of position angle (twisting isophotes), the residual, as a means of amplification, can additionally show (sometimes dramatic) spiral structure. Again we note in Fig. 2 (second row from top) significant position 


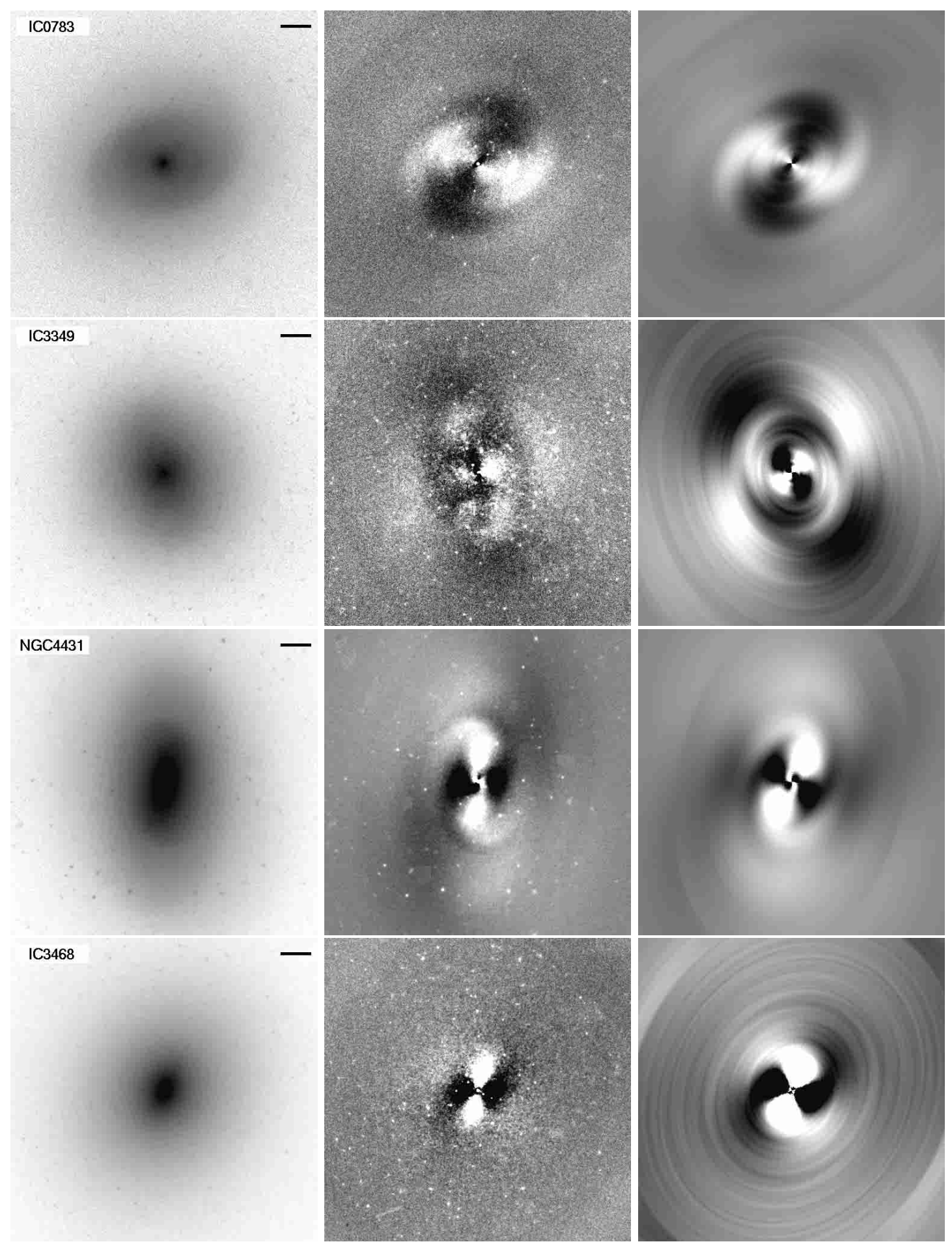

Fig. 1. Left column: $R$-band images of the galaxies. The bar in the upper right corner corresponds to $10^{\prime \prime}(\sim 1 \mathrm{kpc})$. Middle column: residuals obtained by subtracting the mean, deprojected surface brightness profile, corresponding to the first Fourier coefficient $I_{0}$ from the $R$-band image. Right column: representation of the third Fourier coefficient (Fourier map), see text. Image size: 1'.7 × 1'.7. North is up and east to the left.

angle changes in IC 0783 and NGC 4431, where we found traces of spiral arms. As ellipticity changes and twisting isophotes are quite commonly found in spheroidal galaxies, possibly as a result of their intrinsic triaxiality (e.g. Kormendy 1982; Binney \& Merrifield 1998), it is thus clear that hourglasslike and spiral-like features found in residual images of the kind shown in Fig. 1 must not, at face value, be taken as indication for the presence of a true bar or true spiral structure (i.e. disk structure). However, as mentioned in the introduction and as demonstrated in the following section, the residual features in our four dwarfs clearly persist when scrutinized with an unsharp masking method. Their strength makes it difficult to explain these features as mere artifacts produced by such parameter combinations; it is more likely that they indicate the 
IC0783
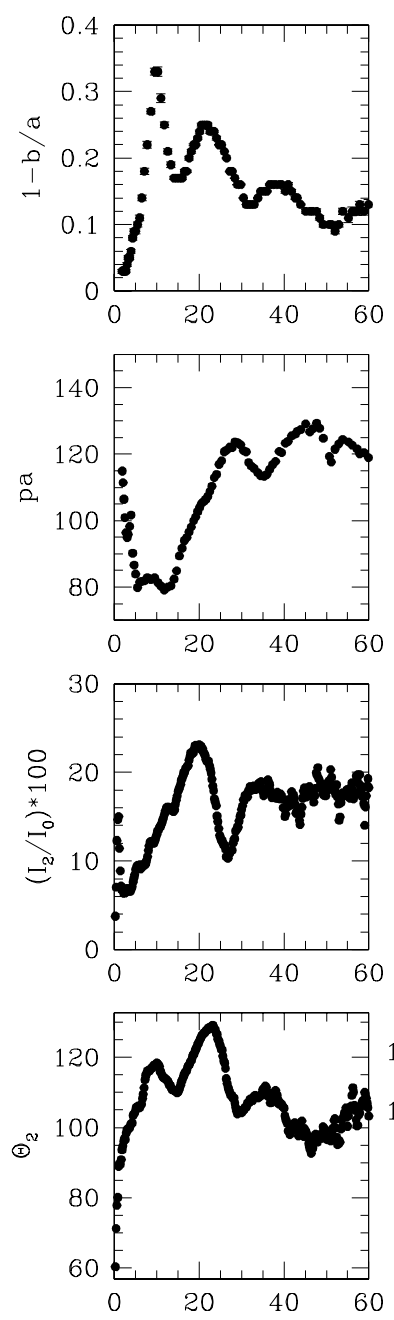

IC 3349
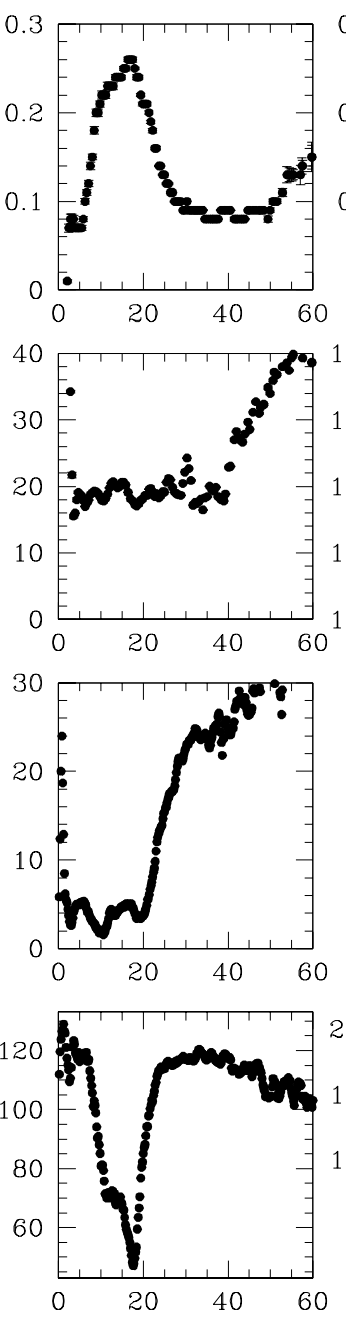

NGC 4431
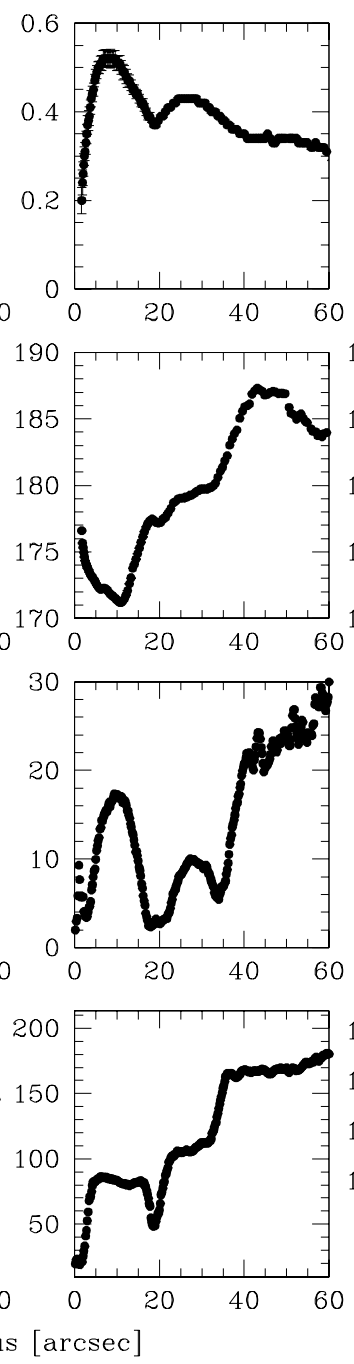

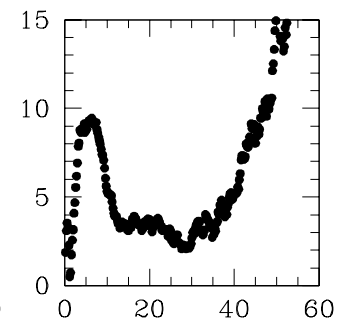

IC 3468
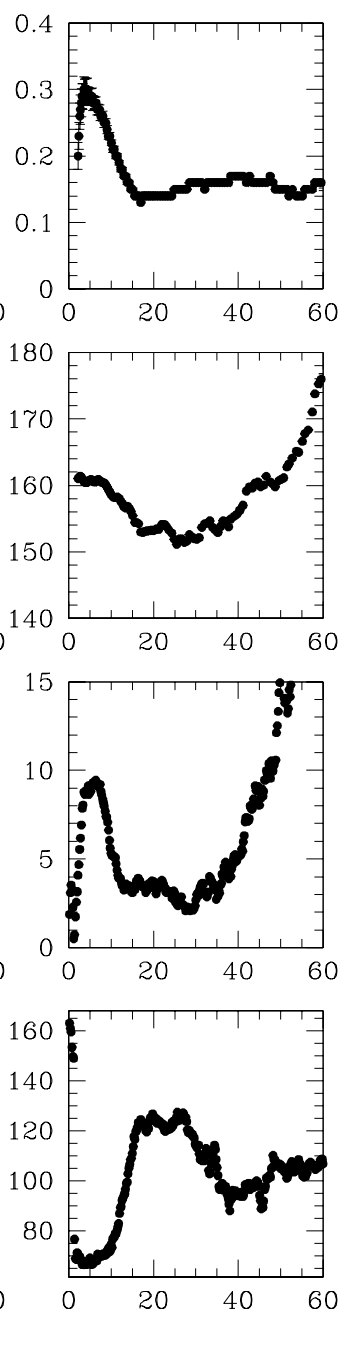

Fig. 2. First row: ellipticity profile; second row: position angle profile. Third row: relative strength, in percent, of the third Fourier coefficient $I_{2}$ relative to $I_{0}$. Fourth row: profile of the phase angle $\Theta_{2}$ of the third Fourier coefficient. All parameters are plotted against the equivalent galactocentric radius.

presence of a real disk (bar and/or spiral) structure that is naturally accompanied by a systematic change in ellipticity and position angle when ellipses are fitted to the surface brightness distribution.

To analyse the residual features found in a more quantitative manner, we then Fourier decomposed the direct images. This technique has frequently been used in the past to quantify non-axisymmetric structures in disk galaxies (Grosbol 1987; Elmegreen et al. 1989; Rix \& Zaritsky 1995; Vera-Villamizar et al. 1998). We used the Fourier expansion in the polar form, with azimuthal angle $\Theta$. To this end we had to determine the centers of the galaxies first. Since we did not measure a considerable offset for any of the nuclei, we derived the centers by fitting a Gaussian to them. The pixel positions could then be expressed in polar coordinates. All isophotes were subsequently deprojected using the ellipticities and position angles from Table 1, and represented by 128 pixels uniformly distributed over each isophote. Finally, the corresponding intensities, $I(r, \Theta)$, were expanded in the Fourier series:

$$
\begin{aligned}
I(r, \Theta) & =I_{0}(r)+I_{1}(r) \cos \left[\Theta-\Theta_{1}(r)\right] \\
& +I_{2}(r) \cos 2\left[\Theta-\Theta_{2}(r)\right]+\ldots
\end{aligned}
$$

The zeroth order Fourier coefficient, $I_{0}$, only depends on the radius and is therefore equivalent to the mean surface brightness of the galaxy. Actually, converting $I_{0}$ in magnitudes and plotting it versus radius yields the mean surface brightness profile. The first order Fourier coefficient, $I_{1}$, characterises the offset of the isophote with respect to the chosen center. Hence, this parameter is a measure of the lopsidedness of a galaxy and the associated phase angle, $\Theta_{1}$, indicates the direction of the offset. It could also be used to measure the offset of the nucleus from the center of the brightness distribution. The next coefficient, $I_{2}$, is associated with even-numbered asymmetries, e.g. structures like bars or an even number of spiral arms. Since the residuals in Fig. 1 show structures of this kind, we plotted the 

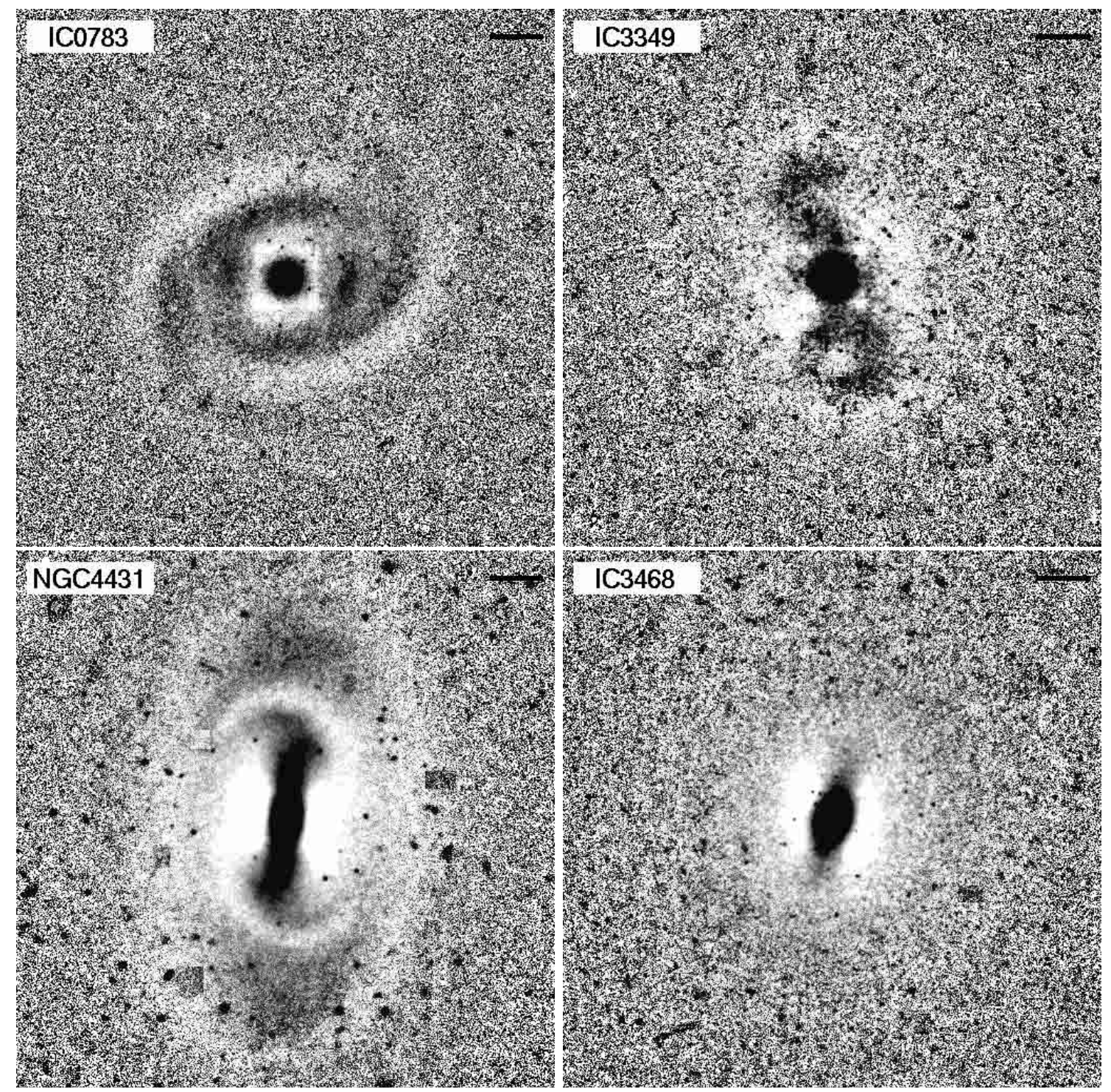

Fig. 3. Result of unsharp masking for the four galaxies. The images are negatives. Size and orientation as in Fig. 1.

relative strength of $I_{2}$, expressed as $\left(I_{2} / I_{0}\right) * 100$, in the third row of Fig. 2 (intensity profile). The values shown therefore indicate the strength of the structures in percent of the underlying luminosity. In the fourth row the corresponding phase angle, $\Theta_{2}$, is shown. This angle points to the position of the signal on the isophote. With respect to the radius it remains constant for a bar, indicating its position angle (e.g. NGC 4431), and rises/decreases linearly for a spiral pattern (as beautifully seen in IC 3328; cf. Jerjen et al. 2000).

However, the interpretation of this phase angle profile is not always so clear. We have therefore developed a method to map the third term of the Fourier expansion, i.e. $I_{2}(r) \cos 2[\Theta-$ $\left.\Theta_{2}(r)\right]$. Since each pixel belongs to an ellipse around the center, we can expand this specific ellipse in the given Fourier series and transform the pixel intensity according to

$I_{x, y}(r, \Theta)=I_{2}(r) \cos 2\left[\Theta_{x, y}-\Theta_{2}(r)\right]$

where $x, y$ are the pixel coordinates. This procedure yields a visual representation of the information contained in the plots of the third and fourth row in Fig. 2. In the following, we denote these representations as "Fourier maps". The results for the sample galaxies are shown in the third column of Fig. 1. The Fourier maps are very similar to the residuals (middle column of Fig. 1), confirming that the structures shown by the residuals are of the symmetric type, giving a strong signal in $I_{2}$.

Let us now go through our sample galaxies, case by case. Considering the direct image and the ellipticity profile, IC 0783 is clearly showing spiral structure. Unfortunately, the residual and the Fourier map, as well as the phase angle profile are not equally revealing. The reason for this might be the existence of an inner ring: its phase angle would be undefined and could cause the observed hump between $10^{\prime \prime}$ and $15^{\prime \prime}$. IC 3349 is rather puzzling. The residual image of this galaxy in Fig. 1 shows an elongated, bar-like structure which is also evident in the ellipticity profile, where it appears as a prominent maximum around $20^{\prime \prime}$, and in the position angle, which is more or less constant in the same range. However, this does not appear in the Fourier parameter plots. The phase angle is strongly 
varying and the intensity profile shows almost no signal in this region. In contrast, NGC 4431 is a clear case. The phase angle remains nearly constant between $4^{\prime \prime}$ and $17^{\prime \prime}$, the $I_{2}$ profile shows a corresponding luminosity excess of $\sim 18 \%$, and the ellipticity profile is considerably peaked: all of this clearly points to the presence of a bar. The case of IC 3468 is similar to this, but on a much smaller scale and with lower intensity. A sharp peak in the ellipticity and intensity profile, along with a constant phase angle are again pointing to a bar, whose length and intensity reach about half the values shown by NGC 4431 .

\section{Unsharp masking}

A well-known method to uncover hidden structures that is independent of the Fourier analysis, and that also has the advantage that no assumptions are required, is unsharp masking. This method, which is also applicable to photographic plates (Malin \& Zealey 1979), was widely used in the search for hidden inner structures in different objects. It was successful in investigating the fine structures in elliptical galaxies (Schweizer \& Ford 1985), dust features in globular clusters (Mendez et al. 1989), and the true nature of bars in disk galaxies (Buta \& Crocker 1993). Recently, Colbert et al. (2001) investigated the morphological differences between elliptical galaxies in different environments using, among other methods, unsharp masking.

The method essentially consists of deconvolving the optical image with an appropriate function. Often a point spread function, like a Gaussian, is used. We chose an even simpler approach: we produced a smoothed image by replacing each pixel intensity with the average intensity in a certain area around that pixel. The optimal size of this area obviously depends on the extension, or scale of the structure to be uncovered. We fixed this size simply by trying different smoothing lengths until the result was satisfactory. Applying the MIDAS task FILTER/SMOOTH, we obtained the best results with a smoothing radius of 30 pixels, corresponding to $6^{\prime \prime}$. Hence, each pixel intensity was set to the average intensity in a square of $31 \times 31$ pixels. The original image was then divided by this smoothed version. The result of this procedure for our four galaxies is shown in Fig. 3.

All four objects show very strong residual features - we will discuss them in detail in the following section. What was guessed before on the basis of "global" residual images and a Fourier analysis now appears with utmost clarity. It is important to note again that no assumptions are required here. We do not have to assume a global ellipticity and position angle in advance, to which the residuals refer - with unsharp masking we determine the residual light with respect to the local environment based on the observed brightness distribution alone.

Whenever residual features are uncovered in otherwise perfectly spheroidal galaxies, the presence of dust has to be considered as a possible cause (dust lanes in the cores of bright ellipticals are quite common; cf., e.g., Binney \& Merrifield 1998). Dust can be detected by its reddening of the colour. However, the $B-R$ colour maps of our galaxies do not show any conspicuous features other than a slight symmetric colour gradient (to be reported elsewhere). The only possible exception is NGC 4431 whose colour map reveals that the bar may be slightly redder than the rest of the galaxy. In particular, IC 3349, where dust could be suspected because the bar-like feature appears somewhat lumpy, is perfectly smooth in colour as well. This means that the residual brightness features reflect density enhancements in the distribution of stars - which of course is the case with true bars and spirals.

\section{Discussion}

The disk nature of IC 0783 has been conjectured before and this object has therefore been classified as dS0 (Sandage \& Binggeli 1984; Binggeli \& Cameron 1991). The possible presence of two spiral arms has already been reported (Barazza et al. 2001; Jerjen et al. 2001). This finding is clearly confirmed by our results. To what kind of central structure these arms are connected is not so evident, however. Since the phase angle is not constant in the central part, the presence of a bar is not very likely. We rather suggest the existence of an inner ring, as mentioned in Sect. 3. The strongest luminosity excess is observed at the locations where the spiral arms start, i.e. the connection points between the ring and the arms. This can be seen in the Fourier maps and also in the intensity profile, which reaches a relative excess of $\sim 24 \%$.

During the refereeing stage of this paper, Simien \& Prugniel (2002) have published a rotation curve of IC 0783 (the authors classify this galaxy as $\mathrm{SAB}(\mathrm{rs}) 0 / \mathrm{a}$ in the de Vaucouleurs system, which is in agreement with our own new classification). Not surprisingly, as judged from the spiral morphology, the galaxy does show rotation, though only with a observed maximal radial velocity of $v_{\max }=22 \mathrm{~km} \mathrm{~s}^{-1}$, or $v_{\text {rot }}=33 \mathrm{~km} \mathrm{~s}^{-1}$ in the plane of the disk, if we assume that the galaxy's apparent ellipticity of 0.25 (Table 1) reflects the disk's inclination only. The observed central velocity dispersion is even a bit larger with $\sigma=36 \mathrm{~km} \mathrm{~s}^{-1}$ (however, the uncertainties, also for the inclination, are considerable).

IC 0783 is situated in a region of rather low density within the Virgo cluster. The distance to the cluster center, i.e. M 87, is quite large. In fact, this galaxy is probably a member of a group around the giant Sbc galaxy M 100. The projected distance between M 100 and IC 0783 is $\sim 100 \mathrm{kpc}$ and the radial heliocentric velocities are $v_{\odot}=1571 \mathrm{~km} \mathrm{~s}^{-1}$ and $v_{\odot}=1293 \mathrm{~km} \mathrm{~s}^{-1}$ for M 100 and IC 0783, respectively (all velocities from the NED). IC 0783 could therefore be bound to M 100. The whole group is believed to have fallen into the cluster recently (Binggeli et al. 1987). Even if the group were falling in for the first time, IC 0783 could already have been stripped of its gas and stopped star formation, in accord with its rather red colour of $B-R=1.34$ and the non-detection of the spiral structure in the colour map.

IC 3349 has been classified as $\mathrm{dE} 1, \mathrm{~N}$; it is very round and has a bright nucleus. The image from unsharp masking reveals an elongated structure almost along the major axis of the galaxy. As mentioned in Sect. 3, the knot lying south-east of the nucleus is caused by a background galaxy. Therefore, the feature is quite symmetric. The best photometric evidence for the structure is provided by the ellipticity profile, which shows a pronounced maximum at $\sim 20^{\prime \prime}$. Despite its weakness (the luminosity excess amounts only to $\sim 5 \%$ ), the structure clearly resembles a bar. Its thick, dumb-bell-like ends are in fact 
reminiscent of the classical $\mathrm{SB}_{3}$ Hubble type. There is even a slight hint for rudimentary spiral/trailing arms as in NGC 4431 (though much less clearly). In any case, the presence of a bar suggests that IC 3349 is a rotating disk galaxy. There is no kinematic data available yet to confirm this. IC 3349 is located in the Virgo M 87 subcluster as well, i.e. in the densest region of the cluster. It is more or less between M 87 and M 86, but closer to the latter, and does not seem to be associated with a giant galaxy.

The most impressive discovery is the beautiful bar in NGC 4431. This galaxy has been classified as dSO before, though without acknowledging the presence of a bar (Sandage \& Binggeli 1984); we now would call it a dSB0/a. Its twisting isophotes have been pointed out by Ryden et al. (1999), where a contour plot of a $V$-band image is shown. Also Gavazzi et al. (2001) observed this galaxy in the $B$ - and $V$-band and performed a profile decomposition.

In the unsharp masked image in Fig. 3 the bar and the trailing arms of NGC 4431 are very striking. The whole morphology is reminiscent of the results of a bar simulation presented by Patsis \& Athanassoula (2000). The gas density distribution plotted in Fig. 5 of that paper shows exactly the same features. In particular, the authors draw attention to the typical " $T$-structure", consisting of the bar, the trailing arms plus short density enhancements on the leading side of the bar. Even the gaps beyond the outer edges of the trailing arms, followed by another pair of denser filaments, are much the same in NGC 4431 and the simulation. Given this similarity, we can assume that the bar in NGC 4431 is rotating clockwise. However, we should also note a difference in size: the bar in the simulation has a length of $\sim 10 \mathrm{kpc}$, whereas the observed one measures only $\sim 3 \mathrm{kpc}$. But just this smallness may provide another interesting aspect: As dwarf galaxies are believed to contain large amounts of dark matter, and as the pattern speed of a bar is governed by the mass distribution via dynamical friction (Debattista \& Sellwood 1998, 2000), objects like NGC 4431 might offer new possibilities to put constraints on the dark matter content of dwarf galaxies.

The pattern speed of the bar in NGC 4431 is still unknown, but its solid body rotation has been measured: the rotation curve determined by Simien \& Prugniel (2002) nicely shows the expected linearity over the scale of the bar. The maximum rotational velocity for NGC 4431 is listed as $v_{\max }=60 \mathrm{~km} \mathrm{~s}^{-1}$. As the galaxy is apparently strongly flattened $(\epsilon=0.38$, Table 1$)$, meaning its disk is strongly inclined, this transforms into a mere $v_{\text {rot }}=76 \mathrm{~km} \mathrm{~s}^{-1}$ in the plane of the disk - barely larger than the measured central velocity dispersion of $\sigma=55 \mathrm{~km} \mathrm{~s}^{-1}$.

NGC 4431 is close to the center of the Virgo cluster; its projected distance to $\mathrm{M} 87$ is $\sim 300 \mathrm{kpc}$. It is therefore located in a rather dense region and belongs to subcluster A (Binggeli et al. 1987). In fact the dwarf-like SBa galaxy NGC 4440 and another dS0 type, NGC 4436, lie within a projected radius of $\sim 40 \mathrm{kpc}$ (see image in Sandage \& Binggeli 1984). However, the radial velocities of these galaxies are quite different. NGC 4431 itself has $v_{\odot}=913 \mathrm{~km} \mathrm{~s}^{-1}$, the closest neighbors (in projection), NGC 4436 and NGC 4440, have $v_{\odot}=1163 \mathrm{~km} \mathrm{~s}^{-1}$ and $v_{\odot}=724 \mathrm{~km} \mathrm{~s}^{-1}$, respectively. A physical grouping of these dwarfs is therefore unlikely.
Finally, a small, bar-like feature is evident in the unsharp masked image of IC 3468. The peak in the ellipticity profile is very narrow and the relative intensity reaches almost $\sim 10 \%$. Note that the galaxy has been classified E1, N instead of dE1, N - precisely on the basis of the high-surface brightness central core that was found to be indicative of a classical (nondwarf) elliptical. It is not clear whether we see a small disk nearly edge-on embedded in an almost round (dwarf) elliptical, or a very short bar embedded in a disk galaxy almost face-on. Judging from the pointed (lemon-shaped) isophotes of the residual feature (as confirmed by a "disky" value of $a_{4} / a \sim 0.01$ in the inner $15^{\prime \prime}$; see below), a disk component within a spheroid would seem more likely. Surprisingly, however, Simien \& Prugniel (2002) were not able to measure any appreciable rotation in IC 3468, listing $v_{\max }<9 \mathrm{~km} \mathrm{~s}^{-1}$ for this galaxy (along the correct position angle of the elongated inner feature). An embedded edge-on disk can therefore be excluded. But a bar in an almost face-on disk is a difficult interpretation as well, because with $\epsilon=0.17$ (Table 1) the deprojected velocity of $v_{\text {rot }}<16 \mathrm{~km} \mathrm{~s}^{-1}$ would still be much smaller than the central velocity dispersion $\sigma=36 \mathrm{~km} \mathrm{~s}^{-1}$. With $v_{\max } / \sigma=$ 0.25 , IC 3468 appears to be a typical anisotropic (non-rotationsupported) dwarf elliptical. One might argue, though, that the maximum of the rotation curve was not reached by Simien $\&$ Prugniel (2002); their measurements indeed cover only the innermost (barred) part of the galaxy. We think the case - for or against the presence of disk structure in this galaxy - is left open. As to its position in the cluster, IC 3468 is situated between the A subcluster, dominated by M 87, and the M 49 subcluster in the south of Virgo and is therefore in a region of rather low density.

We have also done an isophotal analysis for all our Virgo dwarfs observed with the VLT, the results of which will be reported elsewhere (Barazza et al., in preparation). In such an analysis one is looking for systematic deviations of the isophotes from pure ellipses, again by means of a Fourier method. The fourth Fourier coefficient, $a_{4}$, determines whether the isophotes are "disky" $\left(a_{4}>0\right)$ or "boxy" $\left(a_{4}<0\right)$. This parameter is believed to be sensitive to weak disk or bar components in elliptical galaxies, depending on the inclination angle (Carter 1987; Bender \& Möllenhoff 1987; Rix \& White 1990; Ryden et al. 1999). Our disk/bar dwarf candidates do show some correlation with the $a_{4}$ parameter. We have already mentioned the inner diskyness of IC 3468. While IC 0783 does not show a coherent $a_{4}$ signal, NGC 4431 is slightly boxy, as is IC 3349 slightly disky (surprisingly), in the central residual region. However, in general we think the meaning of $a_{4}$ is difficult to interpret; different combinations of components can produce the same $a_{4}$ profile. In particular, disky isophotes need not necessarily mean that there is a true disk component embedded in the galaxy. We are currently exploring the principal possibilities to model $a_{4}$ profiles.

The best evidence for the presence of disk components in dwarf ellipticals or dS0s can be expected to come from kinematic studies. These difficult observations are now underway for a large number of early-type dwarfs in the Fornax and Virgo clusters (de Rijcke et al. 2001; Geha et al. 2001). So far, the majority of dwarfs have turned out to be non-rotating spheroids. 
However, a few are rotation-supported and may plainly be disk galaxies (Simien \& Prugniel 1998). Interestingly, this holds only for Virgo cluster dwarfs; no rotators were found so far among Fornax dEs, nor did any of them exhibit the kind of spiral or bar features reported in the present paper (de Rijcke, private communication).

Our findings support the conjecture that a rather large number of bright, early-type dwarf galaxies, at least in the Virgo cluster, are disks or at least possess a disk component. These systems might formerly have been late-type disk galaxies which, by interactions with the cluster, were transformed to the systems we observe today. Such processes are described for example in the harassment scenario developed by Moore et al. (1998). In this picture, galaxies falling into the cluster lose all of their gas and most probably transform to a spheroid by gravitational interactions with the other cluster members. However, massive late-type galaxies might preserve their disk nature, at least for some time. The galaxies discussed could therefore be transient remnants of the harassment process. Also with respect to this scenario, the Fornax cluster may be a different galaxy environment because it is more isolated in space. The Virgo cluster is surrounded by clouds of late-type galaxies that seem to be fed into the cluster at a constant rate (see, e.g., Binggeli et al. 1987). Perhaps the high frequency of hidden disks in dEs is unique for the Virgo cluster environment.

\section{Summary and conclusions}

Following the discovery of spiral structure in a early-type dwarf galaxy classified as dE, $\mathrm{N}$ by Jerjen et al. (2000), we searched our whole VLT-sample of bright Virgo dEs and dSOs for further photometric disk signatures. The principal search tools applied were (1) looking for inner residual features by subtracting a model image of the galaxy based on its mean surface brightness profile, but with fixed ellipticity and position angle, from the observed image; (2) a Fourier expansion of the galaxy image in polar form, where the lowest order of non-axisymmetry is indicating the presence of a bar or two-armed spiral structure; and (3) unsharp masking, where the original image is divided by an appropriately smoothed one to enhance any local "irregularities". Unsharp masking turned out to be the most reliable method to uncover hidden structures. The first method can give misleading results, if the inherent assumptions on global symmetry, as well as the high sensitivity of the outcome to slightly varying position angles are not taken into account. But once the clear-cut choice by unsharp masking is made, the first two methods are useful to visualize and quantify the symmetric residual structures found.

In addition to IC 3328 (Jerjen et al. 2000), we found photometric traces of a possible disk component in four more earlytype dwarfs out of a sample of 23:

IC 0783: The two spiral arms of this "dS0" galaxy are already evident in the direct optical image. Obviously this is a disk galaxy, which now is also confirmed by the measurement of its rotation (Simien \& Prugniel 2002). The central structure of this galaxy remains unresolved; we think there could be an inner ring.
IC 3349: Fourier analysis and unsharp masking reveal a long and elongated (if only weak) structure in the central part, which we interpret as a bar in a nearly face-on disk. A revised type for this "dE" galaxy would be dSB0.

NGC 4431: The quite strong bar present in this galaxy is the most striking discovery and clearly reveals the disk nature of this dwarf - again nicely confirmed by its measured rotation (Simien \& Prugniel 2002). Besides the bar we clearly note trailing arms and two dense regions on the leading side of the bar. This so-called T-structure is very similar to the results of a simulation presented by Patsis \& Athanassoula (2000). A more fitting type for this "dSO" galaxy would be dSB0/a.

IC 3468: In the very center of this dwarf elliptical we either observe a rather short bar in a nearly face-on disk, or a small disk seen edge-on in a spheroid. Surprisingly, Simien \& Prugniel (2002) found essentially zero rotation along the position angle of this structure, which renders a clear interpretation of what we see impossible at present.

We emphasize that none of these objects is comparable to IC 3328. The weak and uniformly wound spiral structure in this galaxy seems to be truly unique, constituting a particular class of dwarf galaxies; at least we did not find an additional example.

Our findings confirm previous suggestions that a sizeable fraction of all bright early-type dwarfs in the Virgo cluster are disk galaxies. In a possible scenario for their evolution they are former late-type disk galaxies which have been transformed to the systems we observe today during their infall to the cluster. The discovery of more objects of this kind in the Virgo cluster, but also in other clusters, could therefore further constrain possible models for the formation and evolution of early-type galaxies in general.

Acknowledgements. The authors thank Victor Debattista for inspiring discussions. F.D.B and B.B. are grateful to the Swiss National Science Foundation for financial support. This research has made use of the NASA/IPAC Extragalactic Database (NED) which is operated by the Jet Propulsion Laboratory, California Institute of Technology, under contract with the National Aeronautics and Space Administration, as well as NASA's Astrophysical Data System Abstract Service.

\section{References}

Abadi, M. G., Moore, B., \& Bower, R. G. 1999, MNRAS, 308, 947

Barazza, F. D., Jerjen, H., \& Binggeli, B., in preparation

Barazza, F. D., Binggeli, B., \& Jerjen, H. 2001, in Dwarf Galaxies and their Environment, ed. K. S. de Boer, R.-J. Dettmar, \& U. Klein (Shaker Verlag), 243

Bender, R., \& Möllenhoff, C. 1987, A\&A, 177, 71

Binggeli, B., Sandage, A., \& Tammann, G. A. 1985, AJ, 90, 1681

Binggeli, B., Tammann, G. A., \& Sandage, A. 1987, AJ, 94, 251

Binggeli, B., \& Cameron, L. M. 1991, A\&A, 252, 27

Binggeli, B., \& Popescu, C. C. 1995, A\&A, 298, 63

Binney, J., \& Merrifield, M. 1998, Galactic Astronomy (Princeton University Press)

Buta, R., \& Crocker, D. A. 1993, AJ, 106, 939

Carter, D. 1987, ApJ, 312, 514

Colbert, J. W., Mulchaey, J. S., \& Zabludoff, A. I. 2001, AJ, 121, 808

Debattista, V. P., \& Sellwood, J. A. 1998, ApJ, 493, L5

Debattista, V. P., \& Sellwood, J. A. 2000, ApJ, 543, 704 
De Rijcke, S., Dejonghe H., Zeilinger, W. W., \& Hau, G. K. T. 2001, ApJ, 559, 21

Elmegreen, B. G., Elmegreen, D. M., \& Seiden, P. 1989, ApJ, 343, 602

Ferguson, H. C., \& Binggeli, B. 1994, A\&AR, 6, 67

Gavazzi, G., Zibetti, S., Boselli, A., et al. 2001, A\&A, 372, 29

Geha, M., Guhathakurta, P., \& van der Marel, R. 2001, in The Shapes of Galaxies and their Halos, ed. P. Natarjan (Yale Cosmology Workshop)

Grosbol, P. 1987, in Selected Topics on Data Analysis in Astronomy, ed. L. Scarsi, V. DiGesu, \& P. Crane (World Scientific), 57

Jerjen, H., Binggeli, B., \& Kalnajs, A. 2000, A\&A, 358, 845

Jerjen, H., Kalnajs, A., \& Binggeli, B. 2001, in Galaxy Disks and Disk Galaxies, ed. G. José, S. J. Funes, \& Enrico Maria Corsini, ASP Conf. Ser., 230, 239

Jerjen, H., Binggeli, B., \& Barazza, F. D., in preparation

Kormendy, J. 1982, in Morphology and Dynamics of Galaxies, 12th Advanced Saas-Fee course, ed. J. Binney, J. Kormendy, \& S. D. M. White (Swiss Society for Astrophysics and Astronomy), 149
Malin, D. F., \& Zealey, W. J. 1979, S\&T, 57, 354

Mendez, M., Orsatti, A. M., \& Forte, J. C. 1989, ApJ, 338, 136

Moore, B., Lake, G., \& Katz, N. 1998, ApJ, 495, 139

Nieto, J.-L., Bender, R., Poulain, P., \& Surma, P. 1992, A\&A, 257, 97

Patsis, P. A., \& Athanassoula, E. 2000, A\&A, 358, 45

Rix, H.-W., \& White, S. D. M. 1990, ApJ, 362, 52

Rix, H.-W., \& Zaritsky, D. 1995, ApJ, 447, 82

Ryden, B. S., \& Terndrup, D. M. 1994, ApJ, 425, 43

Ryden, B. S., Terndrup, D. M., Pogge, R. W., \& Lauer, T. R. 1999, ApJ, 517, 650

Sandage, A., \& Binggeli, B. 1984, AJ, 89, 919

Schweizer, F., \& Ford, W. K. Jr. 1985, in New Aspects of Galaxy Photometry, ed. J. L. Nieto (Springer Verlag), 145

Simien, F., \& Prugniel, Ph. 2002, A\&A, 384, 371

Vera-Villamizar, N., Dottori, H., de Carvalho, R., \& Puerari, I. 1998, in Focal Points in Latin American Astronomy, ed. A. Aguilar, \& A. Carraminana (Rev. Mex. Astron. Astrofis. S. Conf.) 